\title{
A cultura matemática mobilizada por licenciandos no contexto de uma disciplina de análise real
}

\author{
Diego Matos \\ Colégio Pedro II (CPII) \\ diego_matos_p@hotmail.com
}

\section{Victor Giraldo}

Universidade Federal do Rio de Janeiro (UFRJ)

victor.giraldo@gmail.com

\section{Wellerson Quintaneiro}

Centro Federal de Educação Tecnológica (CEFET-RJ)

profmatwellerson@gmail.com

\begin{abstract}
Resumo
A literatura de pesquisa em Educação Matemática tem destacado uma desarticulação entre a licenciatura e a prática docente na educação básica. Nesse contexto, o presente trabalho traz resultados de uma pesquisa cujos objetivos eram: identificar aspectos da cultura matemática mobilizada por licenciandos no contexto de um curso de Análise Real; observar possíveis relações entre essa cultura e a construção de saberes docentes. Os dados empíricos foram produzidos a partir de questionários, tarefas envolvendo o Teorema do Valor Intermediário e entrevistas semiestruturadas. Os resultados evidenciaram aspectos de uma cultura matemática associada à percepção hierárquica sobre matemática escolar e matemática acadêmica; manifestando-se, sobretudo, por meio da valorização do rigor em detrimento de aspectos considerados "menos formais", como representações gráficas ou argumentações sem escrita simbólica. Essa cultura esteve associada a critérios de legitimação da argumentação matemática, inclusive em tarefas privilegiando situações de ensino na educação básica, indicando relação entre a cultura matemática observada e a construção de saberes docentes.
\end{abstract}

Palavras-chave: Cultura matemática. Formação de professores. Saberes docentes.

\section{Mathematical culture driven by prospective teachers in the context of a real analysis course}

\begin{abstract}
Research literature in Mathematics Education has been highlighting a gap between pre-service teachers' education and their future activities in the classroom. This paper presents results from a research aiming at: identifying aspects of a mathematical culture driven by prospective teachers in the context of a Real Analyses module; mapping out possible relations between this culture and the construction of teachers' knowledge. Empirical data were generated from written questionnaires, tasks involving the Intermediate Value Theorem, and post-task semi-structured interviews. Results reveal aspects of a mathematical culture associated with a hierarchical perception about School
\end{abstract}


Mathematics and Academic Mathematics, which arose mostly through the value assigned to rigor sometimes related with steps and symbols associated by the participants with mathematical formal logic - to the detriment of aspects regarded as "less formal", such as graphical representations, or arguments that did not include explicit symbolic writing. Thus, our data suggests that this culture is related with criteria for legimation of mathematical arguing, including in the case of the tasks that focus on school teaching situations. Such results point out to relationships between this mathematical culture and the construction of teachers' knowledge.

Keywords: Mathematical culture. Teachers' education. Teachers' knowledge.

\section{Introdução}

Saberes de conteúdo para o ensino e formação inicial do professor de matemática vêm sendo largamente debatidos na literatura de pesquisa em Educação Matemática. No Brasil, diversos pesquisadores (FIORENTINI; OLIVEIRA, 2013; MOREIRA; FERREIRA, 2013) têm refletido sobre o lugar da matemática na formação inicial e apontado que a Licenciatura em matemática se encontra desarticulada da futura prática docente. Embora os cursos de Licenciatura tenham por objetivo legal formar professores para atuar na educação básica, a formação matemática acadêmica, em geral, evidencia a perspectiva formal da matemática e estabelece poucas conexões com a matemática ensinada na escola.

Fiorentini (1995) destaca que, após 1950, o ensino de matemática no Brasil passou por diversas mudanças curriculares em virtude do engajamento de matemáticos e de professores no movimento internacional conhecido como Matemática Moderna, que promoveu um retorno ao formalismo matemático fundamentado em teoria de conjuntos e em estruturas algébricas, enfatizando os padrões de rigor da matemática acadêmica no uso da linguagem formal. A influência dessa tendência internacional fez com que o estilo formalista penetrasse gradualmente em diversos níveis de ensino de matemática, de modo que o modelo de apresentação definição-teoremademonstração se tornou paradigma de exposição dominante no ensino superior da disciplina (DAVIS; HERSH, 2013).

Tais reflexões nos levam a questionar se os cursos de Licenciatura têm privilegiado uma forma específica de fazer matemática, dentre tantas outras, o que pode estar contribuindo para uma insuficiência em atingir os objetivos estabelecidos para a própria aprendizagem dos conteúdos, como para a construção de saberes docentes e de suas relações com a prática. Entendemos que a valorização exclusiva do aspecto formal da Matemática pode evidenciar uma visão limitada sobre sua natureza. Particularmente em relação ao professor, alinhamo-nos com Davis e Renert (2009) no entendimento de que o conhecimento matemático desse profissional revela-se extenso e dinâmico, 
manifestando-se de diversas formas em termos de uma disposição participativa em um domínio de conhecimento em evolução.

A escolha pela disciplina de Análise Real como contexto de pesquisa da dissertação aqui relatada permite abordar essa problemática a partir da observação das impressões do licenciando sobre a matemática formal, no âmbito de sua formação, e verificar como ele relaciona essas impressões com as expectativas sobre sua futura prática docente. Destacamos que, embora o curso de Análise forneça o contexto da investigação, este não é nosso objeto de análise. O objeto desta pesquisa são os licenciandos, com a observação de aspectos culturais e de saberes docentes em seus discursos. O contexto da investigação se estabeleceu na medida em que diversos tópicos da educação básica - como números reais e funções - apresentam relações com conteúdos do curso de Análise Real. Portanto, diferentes questões matemáticas com vistas ao ensino evidenciam relação com a disciplina que os participantes estavam cursando.

\section{Conhecimento Matemático para o Ensino e Cultura Matemática}

A falta de articulação entre escola e universidade nos cursos de formação inicial de professores não é recente, tampouco restrita ao contexto brasileiro. Em 1908, Felix Klein já denunciava uma dupla descontinuidade nesses cursos, à qual se referiu como "alienação entre escola e universidade". Segundo o autor, ao iniciar sua formação universitária, o estudante não identifica relações entre a matemática apresentada na universidade e aquela aprendida anteriormente na educação básica; e, ao retornar à escola como professor, não estabelece relações entre a matemática que ensina e aquela estudada no curso de graduação. Isso pode levar o professor a desconsiderar o curso de graduação e a reproduzir, em sua prática docente, as mesmas abordagens vivenciadas como aluno da escola básica.

A partir do entendimento da matemática como um corpo orgânico, Klein se refere à percepção hierárquica entre matemática elementar e matemática superior como um obstáculo a ser vencido, uma vez que as considera como facetas de igual importância da Matemática. Na concepção de Klein, a matemática elementar não corresponde a uma matemática "facilitada" ou "mais simples", mas sim às partes essenciais que sustentam e estruturam a matemática como ciência (RANGEL et al, 2015). Para o autor, o conhecimento matemático para o ensino deve incluir uma visão abrangente que permita ao professor observar a matemática elementar de um ponto de vista superior, isto é, uma visão panorâmica das partes estruturantes da Matemática (KILPATRICK, 2008).

Essas ideias de Klein têm paralelos com pesquisas mais recentes sobre o conhecimento matemático do professor. Shulman (1986) define conhecimento pedagógico de conteúdo como um 
domínio especial do conhecimento do professor que abrange os aspectos do conteúdo que o fazem ensinável a outros. Assim, o conhecimento pedagógico de conteúdo transcende o conhecimento sobre o conteúdo per se, abrangendo o conteúdo em sua dimensão pedagógica, um saber sobre o conteúdo para o ensino. Ball et al (2008) reconhecem a contribuição do trabalho de Shulman para a compreensão da profissionalização da docência, considerando suas especificidades e entendendo-a como um campo profissional com saberes próprios. Esses autores propõem um desdobramento das ideias de Shulman a partir de uma variável central na sala de aula: a dinâmica de ensino, considerando as interações nesse cenário. Assim, propõem a noção de conhecimento matemático para o ensino, que corresponde ao conhecimento matemático que professores necessitam na ação de sua prática.

Shulman (1986) e Ball et al (2008) oferecem reflexões importantes sobre a especificidade do saber de conteúdo para o ensino. Porém, esses trabalhos dão pouco destaque à contribuição de práticas sociais para a construção de saberes profissionais docentes - o que nos parece fundamental, sob a perspectiva de Davis e Renert (2009) sobre o saber do professor como um domínio dinâmico em permanente transformação, emergente da participação e da prática, e não como um conjunto fixo de itens, determinado a priori, a ser adquirido pelo professor para o bom exercício da profissão.

Nesse sentido, no âmbito de nossa investigação, entendemos que a atividade matemática deve ser observada mediante um conjunto de práticas sociais e culturais que são determinantes para a constituição de uma cultura matemática mobilizada por uma comunidade. Miguel e Vilela (2008) afirmam que, ao considerarmos as práticas de mobilização de cultura matemática, a Matemática deixa de ser apenas um corpo homogêneo de conhecimento e atinge uma dimensão plural, em um conjunto de práticas sociais das quais participam diversos atores - alunos, professores, matemáticos e todos que estão envolvidos nessa atividade.

[...] Quando falamos em processos de mobilização de cultura matemática, deixamo-nos de nos referir à matemática como um corpo homogêneo e universal de conhecimentos e passamos a falar em matemáticas no plural. E tais matemáticas passam a ser vistas como aspectos de atividades humanas realizadas com base em um conjunto de práticas sociais, tais como aquelas realizadas pelos matemáticos profissionais, pelos professores de matemática, pelas diferentes comunidades constituídas com base em vínculos profissionais, bem como pelas pessoas em geral em suas atividades cotidianas. (MIGUEL; VILELA, 2008, p. 112)

Segundo esse olhar, produzir, ensinar e aprender matemática não podem ser reduzidos exclusivamente à dimensão formal da disciplina, especialmente na formação inicial do professor e em sua prática profissional. Sob essa perspectiva, os saberes docentes não são construídos somente a partir de questões didático-pedagógicas ou da reflexão conceitual sobre o conteúdo. Entendemos 
que esses saberes são dinâmicos, emergentes e indissociáveis do contexto das práticas sociais e culturais mobilizadas ao longo da formação inicial.

\section{Percurso Metodológico}

Visando contribuir com a compreensão sobre as práticas sociais e culturais mobilizadas na Licenciatura, este artigo relata uma pesquisa que buscou investigar: Quais aspectos emergem do discurso $^{1}$ de licenciandos sobre suas concepções relacionadas à natureza da Matemática especificamente no que tange a práticas sociais e culturais observadas no contexto de uma disciplina de Análise Real - e de que maneira tais aspectos se articulam com a construção de saberes matemáticos para o ensino?

A pesquisa de campo foi realizada em uma turma de Análise Real ministrada no curso noturno de Matemática de uma universidade pública do Rio de Janeiro. Para a coleta de dados, foram empregados três instrumentos, que demarcaram as etapas da pesquisa descritas a seguir.

\section{Etapa 1: Questionário}

Esta etapa foi composta por um conjunto de sete questões sobre a trajetória acadêmica dos participantes e sobre o papel da disciplina de Análise em sua formação. O objetivo desse instrumento era traçar um perfil de cada participante e mapear suas impressões sobre a disciplina de Análise no contexto de sua própria formação.

\section{Etapa 2: Tarefas}

Foram propostas três tarefas abordando o Teorema do Valor Intermediário, no contexto de um curso de Análise, além de uma aplicação na matemática escolar. O desenho metodológico das tarefas foi inspirado no trabalho de Biza, Nardi e Zachariades (2007). Nas tarefas 1 e 3, além de resolver a questão proposta, os participantes deveriam: (1) descrever os objetivos da questão; (2) avaliar resoluções (fictícias) de alunos; e (3) indicar o feedback que seria dado a esses alunos.

As tarefas (cujos enunciados são apresentados em anexo) visam verificar se (e como) havia relações entre a forma como os participantes observavam a matemática formal e suas expectativas sobre a futura prática docente. As tarefas foram respondidas individualmente e por escrito.

\footnotetext{
${ }^{1}$ Entendemos como discurso não só o que é expresso pela fala, mas também por registros escritos ou qualquer outra manifestação comunicativa, como ações de hesitação ou expressão de dúvida que podem ter sido apontadas em nossas anotações de campo.
} 


\section{Etapa 3: Entrevistas}

Foram realizadas entrevistas semiestruturadas, cujo roteiro foi elaborado com base em uma análise preliminar dos resultados das etapas anteriores. O objetivo era esclarecer aspectos específicos e aprofundar a interpretação dessas respostas. As entrevistas duraram cerca de uma hora, foram áudio-gravadas e transcritas. A figura 1, ao final da seção, ilustra o percurso metodológico da pesquisa.

\section{Participantes}

Três alunos do curso de Licenciatura em Matemática participaram do estudo, identificados aqui por: Alexandre, Jorge e Rodrigo. Alexandre e Rodrigo cursavam Licenciatura e Bacharelado, enquanto Jorge era aluno apenas de Licenciatura. Jorge e Rodrigo cursavam Análise pela $3^{\text {a }}$ vez, enquanto Rodrigo fazia a disciplina pela $1^{\mathrm{a}}$ vez. Alexandre e Jorge não tinham experiência formal como professores do ensino básico, enquanto Rodrigo, egresso de uma graduação em Física Médica, já havia lecionado matemática e física em pré-vestibulares comunitários.

Figura 1 - Percurso metodológico.

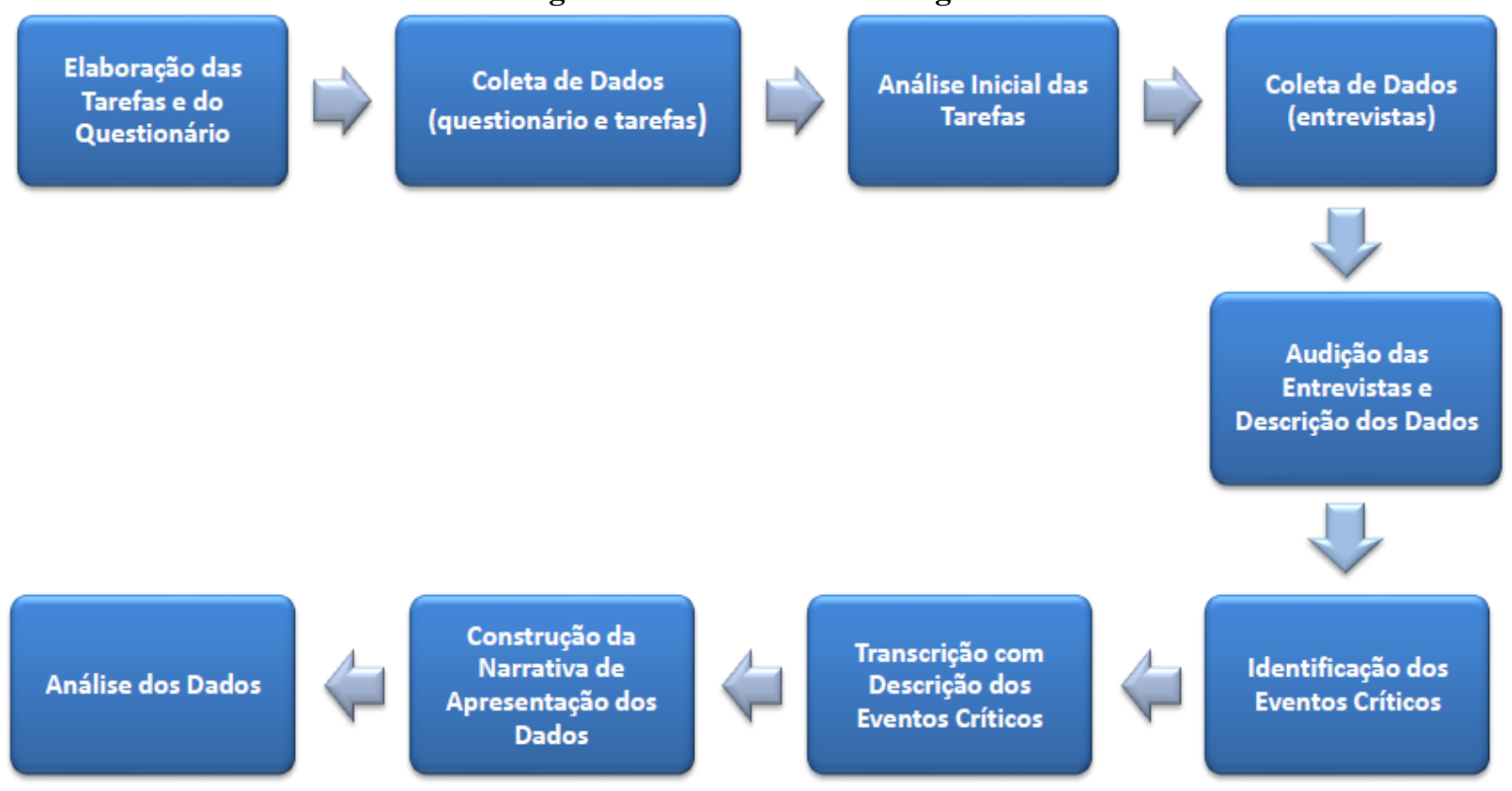

Fonte: Elaborada pelos autores.

\section{Resultados}

Discutimos, a seguir, alguns resultados identificados a partir da análise das respostas dos participantes às tarefas e de aspectos de seu discurso nas entrevistas. Esses resultados permitiram identificar aspectos de uma cultura matemática, no sentido de Miguel e Vilela (2008), mobilizados 
pelos participantes. Destacamos que o reconhecimento de aspectos culturais emergiu, explicitamente, do discurso do participante Rodrigo, quando usou o termo "cultura" ao refletir sobre a importância do curso de Análise na Licenciatura. Na ocasião, Rodrigo se referiu ao curso de Análise como um elemento que condiciona a formação de uma "cultura diferenciada" pelo professor.

Sem Análise a pessoa não pode dizer que é matemático. Análise é o filé mignon da Matemática. É onde estão as ideias. Tudo é um número real. Me diz uma grandeza que não seja um número real! [inquietação] Eu acho que ele vai ser professor de Matemática, ele tem que ter uma cultura diferenciada. (RODRIGO, grifo nosso)

Em nossa interpretação, a referência ao termo "cultura" por Rodrigo evidencia a visão de que o professor é formado a partir de uma cultura matemática mobilizada na Licenciatura. A cultura citada por Rodrigo está articulada a uma percepção hierárquica sobre a Matemática, segundo a qual a disciplina de Análise é vista como algo nobre, uma vez que representa "o filé mignon da Matemática”. Essa percepção hierárquica, caracterizada pela valorização do aspecto formal da matemática, emerge em diversos momentos no discurso dos participantes. Note-se que aqui a percepção hierárquica extrapola os conteúdos, estando também atrelada ao sujeito, que só atinge determinado patamar no topo da hierarquia (só se torna matemático) com o "filé mignon da Matemática": a Análise.

Neste artigo, apresentaremos os resultados da pesquisa em três eixos de análise, que descrevem características de uma prática cultural mobilizada pelos participantes: percepção hierárquica sobre a matemática; critérios de legitimação de argumentação matemática; construção de saberes docentes.

\section{Percepção Hierárquica sobre a Matemática}

Identificamos a atribuição de uma hierarquia entre a matemática apresentada na escola e na universidade, que tem paralelos com a percepção hierárquica entre matemática elementar e matemática superior, descrita por Klein (2004) como um obstáculo a ser vencido. Nesse cenário, os participantes valorizaram a matemática acadêmica, situando-a no topo da hierarquia idealizada, em detrimento da matemática escolar. Relatamos, a seguir, um episódio que consideramos representativo para este eixo de análise, no qual a percepção hierárquica é evidente quando o participante Jorge afirma que "no ensino superior, as questões são mais amplas, mais profundas". De acordo com a percepção manifestada pelo participante, a matemática escolar está em um patamar inferior, uma vez que, no ensino básico, "dificilmente tem questões que te levam para temas que são muito de ponta”. 
No ensino superior, as questões são mais amplas, mais profundas. Você trata de coisas que, muitas vezes, são muito difíceis. No ensino básico, já são temas que são mais solidificados. [...] Agora, a diferença para o ensino superior sempre é essa, porque, mesmo o professor doutor, chefe da cadeira, diretor do departamento, ele olha para um assunto e diz: não entendi o que você fez. Isso se acontece no ensino médio, é por desconhecimento do professor sobre aquele assunto. Mas, é um assunto que ele recorre e pode aprender de um dia para o outro e trazer para o aluno. Acho que existe essa diferença. (JORGE)

Nota-se que essa fala está na direção das críticas feitas pela literatura citada, como Shulman (1986) e Ball et al (2008), pois aponta contrariamente à especificidade do conhecimento de conteúdo para o ensino, quanto à complexidade e ao dinamismo (Davis; Renert, 2009) da prática docente. Além disso, segundo nossa análise, a percepção hierárquica manifestada por Jorge vai além da dimensão do conteúdo, atingindo também um juízo sobre os sujeitos. Jorge atribui que a ausência de retorno ao aluno sobre a matéria se deve a um "desconhecimento", no caso do professor da escola básica; e à complexidade do conteúdo, no caso do professor da universidade. Portanto, o participante julga, a priori, essa ausência como uma deficiência do indivíduo, no caso do professor da escola básica; e como uma qualidade do conteúdo, no caso do professor da universidade - o que, ao mesmo tempo, desqualifica o professor da escola em relação ao professor universitário (até o enaltecendo como "professor doutor”), e valoriza o conteúdo matemático da universidade em relação à matemática escolar.

Consideramos que essa percepção hierárquica esteja relacionada a uma prática cultural mobilizada pelos participantes, concebida a partir da perspectiva formal da matemática, na qual o conhecimento matemático apresentado na Licenciatura é valorizado em detrimento do conhecimento matemático escolar. Já que o conhecimento matemático escolar é simples e pode ser entendido "de um dia para o outro", como afirma o participante, faz muito mais sentido que a Licenciatura se volte para temas complexos. Nesse contexto, essa cultura matemática parece nortear o licenciando na busca de uma identidade cultural que o permita se reconhecer como pertencente ao grupo de matemáticos imersos nessa cultura, como manifestado na fala do participante Rodrigo.

Eu acho que o professor de matemática tem que saber uma demonstração, um teorema. Ele tem a lógica não só como ferramenta, mas como fim. [...] Acho que é de fundamental importância para o professor. Eu não me considero um professor de Matemática sem passar em Análise. (RODRIGO)

Notamos que, na visão de Rodrigo, o curso de Análise é o elemento que identifica um professor de Matemática. Assim, saber Análise é o fator essencial que determina a identidade cultural do professor de matemática. Usamos o termo identidade por considerar que o participante tenta se adequar à cultura matemática observada no curso de Análise em busca de um reconhecimento profissional que o defina como professor de matemática: só pode ser reconhecido como esse profissional aquele que está imerso nessa cultura, que utiliza o rigor e o formalismo 
como parâmetro de valorização em suas argumentações, que "tem a lógica não só como ferramenta, mas como fim".

\section{Critérios de Legitimação de Argumentação Matemática}

Destacamos aqui que a visão dos participantes sobre a matemática parece influenciar seus critérios de legitimação de uma argumentação. Por vezes, eles desqualificaram a utilização de representações gráficas e apontaram a argumentação formal como a maneira correta de apresentar a matemática. Os critérios de validade apresentados pelos sujeitos estiveram associados à percepção hierárquica identificada na subseção anterior, na qual a apresentação formal dos conteúdos do ensino superior era enaltecida em detrimento de outras formas de apresentação matemática.

Outras formas de exposição matemática, como representações gráficas, foram consideradas "rudimentares" e tiveram sua legitimidade contestada, como observado na argumentação de Rodrigo (Figura 2), em resposta à Tarefa 2. Destaca-se que, nessa tarefa, um contraexemplo gráfico seria suficiente para justificar a invalidade das afirmações falsas.

Figura 2 - Argumentação de Rodrigo na Tarefas 2.

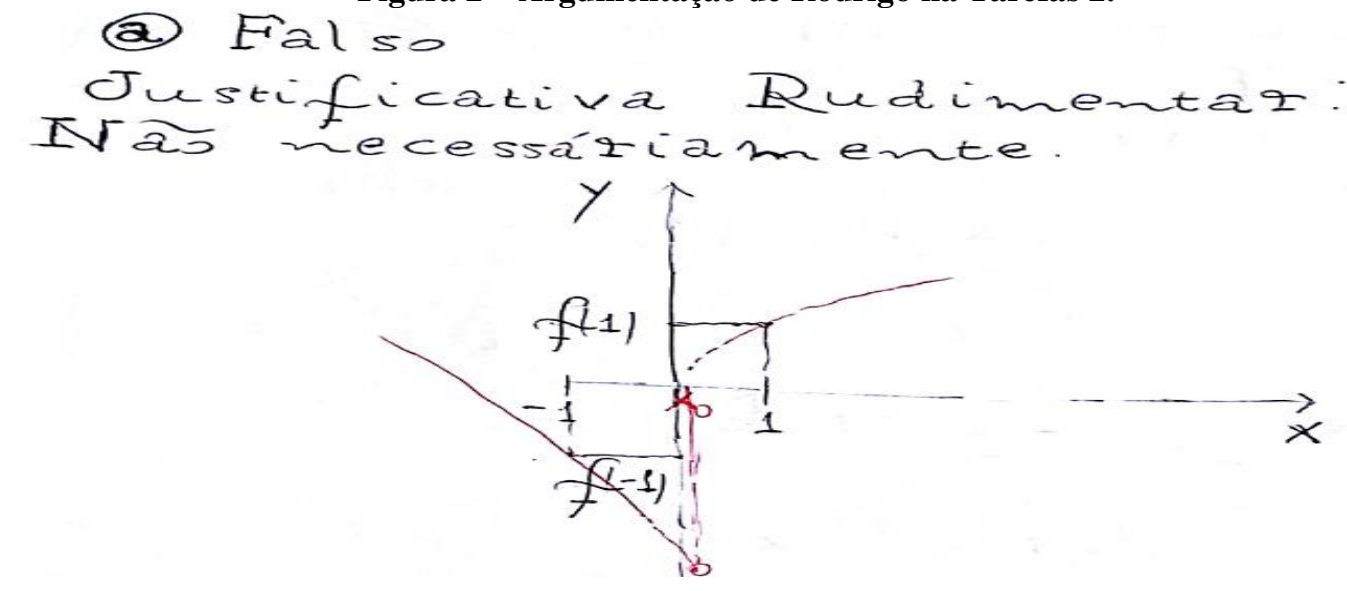

Fonte: Fragmento de pesquisa.

Rodrigo desqualifica sua própria justificativa classificando-a como "rudimentar". Na entrevista, ao argumentar sobre o uso desse termo, afirma que se espera de um aluno de Análise a utilização de uma linguagem simbólica, ao invés de gráficos.

Para alguém que tem uma certa familiaridade com a Análise, eu acho que já demonstra mais os teoremas e usa mais uma linguagem simbólica, ao invés de ficar fazendo os gráficos. [...] O ideal para um matemático seria a lógica pura. Os símbolos e a lógica pura. Para um matemático. Mas, no meu caso, eu ainda não cheguei nesse patamar. (RODRIGO)

Verificamos que, para Rodrigo, a ausência de uma escrita simbólica torna uma argumentação rudimentar. Em nossa análise, essa valorização da forma de uma argumentação 


\section{em detrimento de seu conteúdo foi determinante para ele desqualificar uma ideia matemática}

importante. Em alguns momentos da entrevista, a percepção hierárquica de Rodrigo sobre a matemática fez-se muito presente no uso de termos como "alcançar", "ainda não cheguei nesse patamar", "eu não tenho esse nível”. Interpretamos que a percepção hierárquica de Rodrigo teve implicações diretas no que ele considera legítimo em uma argumentação matemática.

\section{Construção de Saberes Docentes}

Retomemos aqui a fala na qual Jorge afirma que, de modo diferente da graduação, não saber dar retorno sobre uma questão "no ensino médio, é por desconhecimento do professor sobre aquele assunto. Mas, é um assunto que ele recorre e pode aprender de um dia para o outro e trazer para o aluno". Nota-se que essa fala está na direção das críticas feitas pela literatura citada, como Shulman (1986) e Ball et al (2008), pois esta desconsidera a especificidade do conhecimento de conteúdo para o ensino, reduzindo-o ao conhecimento comum de conteúdo, que supostamente pode ser aprendido "de um dia para o outro".

Traçando um paralelo com as ideias desses autores, identificamos possíveis relações entre a concepção formalista identificada nos eixos de análise anteriores e a construção de saberes docentes do licenciando na formação inicial. Os desdobramentos das concepções dos participantes sobre a matemática influenciaram a análise e a legitimação de soluções de alunos na tarefa 1, cujo contexto é a matemática escolar. A primeira demonstração apresentada (Ana) era baseada em uma argumentação gráfica que considerava a aplicação do Teorema do Valor Intermediário, enquanto a segunda (Carlos) utilizava o artifício algébrico da pesquisa de raízes racionais.

Embora ambas as demonstrações apresentassem erros, o participante Alexandre identificou apenas a imprecisão na solução de Ana e reproduziu uma argumentação semelhante à solução de Carlos, que não era matematicamente genérica, uma vez que desconsiderava o caso de zeros irracionais. Assim, Alexandre valorizou uma forma de apresentação matemática, considerada por ele "mais formal”, por apresentar uma escrita simbólica sem representações gráficas - mesmo em detrimento da correção matemática das demonstrações. Na entrevista realizada após a tarefa, quando questionado se sua solução era suficiente para encontrar todos os zeros da função, Alexandre identificou o equívoco. Em seguida, destacou que a estética da escrita do aluno Carlos aparentou que seus argumentos estavam corretos e o induziu a reproduzir sua solução na mesma direção.

[...] aqui ele só iria encontrar os zeros racionais. Agora, depois que eu pensei nisso. Só ia encontrar os zeros racionais, não os irracionais. [...] Mas, talvez, na hora eu me deixei levar por achar que ele tinha verificado certinho e, na de Ana, eu consegui perceber mais a falha. [...] A escrita dele pareceu muito bacana. A escrita 
dele me mostrava que ele estava mais consciente do que estava colocando ali do que Ana. (ALEXANDRE)

Este trecho ilustra relações entre a cultura matemática mobilizada pelo participante e a construção de seu conhecimento de conteúdo. Na sequência deste episódio, Alexandre demonstra que, possivelmente, essa cultura que valoriza a forma de uma argumentação em detrimento de seus significados pode ter reflexos na construção de seus saberes de conteúdo para o ensino. Segundo Alexandre, o professor fica mais atento ao erro quando analisa uma argumentação mais informal.

[...] o professor em si ia ficar mais atento. Se você vê uma linguagem muito informal assim, fugindo do rigor matemático, da linguagem, deve ficar muito mais atento ao erro do que perceber um erro em uma demonstração que parece que vem muito certa, mas, de repente, tem um ponto que ele não usou. (ALEXANDRE)

Em nossa análise, esses aspectos condicionaram os critérios de validade matemática adotados pelo participante ao avaliar a solução de um aluno do ensino básico, uma vez que uma argumentação informal é associada a uma solução que apresenta uma chance maior de conter erros e, por esse motivo, o professor deve estar mais atento à sua avaliação.

\section{Considerações Finais}

Os resultados da pesquisa indicam que os participantes apresentavam uma percepção hierárquica sobre a matemática, concebida a partir de sua perspectiva formal, em que a matemática acadêmica é enaltecida em detrimento da matemática escolar, e, ainda, que tal hierarquia se relacionava com os critérios de validação de argumentos matemáticos. Considerando que as tarefas incluíam análise de soluções de alunos da escola básica, tais critérios podem ainda ter ecos em saberes de conteúdo para o ensino. Também observamos uma prevalência de discursos que desconsideravam a especificidade do conhecimento matemático para o ensino.

Em diversos momentos, os participantes expressaram visões que têm paralelos com a percepção hierárquica denunciada por Klein (2004). Além disso, a hierarquia expressa por eles não se referia, apenas, ao conhecimento matemático, mas se estendia também às figuras dos professores da universidade e da escola básica. Assim, tais questões hierárquicas indicaram forte relação com a percepção dos licenciandos sobre a construção de uma identidade docente.

Os resultados desta pesquisa permitiram descrevermos características de uma prática cultural mobilizada pelos participantes, caracterizada por uma percepção hierárquica que valoriza o rigor da matemática acadêmica. Nesse contexto, posicionar-se nessa hierarquia como aluno da Licenciatura, ou como futuro professor da educação básica, passava por construir uma identidade cultural que possibilitasse reconhecer-se como participante dessa cultura matemática. Consideramos, finalmente, que a identificação dessa cultura nos fornece subsídios importantes para 
repensarmos o ensino de matemática na universidade e, em particular, os modelos para a formação inicial de professores de matemática.

\section{Referências}

BALL, D.; THAMES, M.; PHELPS, G. Content knowledge for teaching: What makes it special? Journal of Teacher Education, v. 59, n. 5, p. 389-407, 2008.

BIZA, I.; NARDI, E.; ZACHARIADES, T. Using tasks to explore teacher knowledge in situationspecific contexts. Journal of Mathematics Teacher Education, v. 10, n. 4-6, p. 301-309, 2007.

DAVIS, B; RENERT, M. Mathematics for teaching as shared, dynamics participation. Learning of Mathematics, v. 29, n, 3, p. 37-43, 2009.

DAVIS, P.; HERSH, R. A Experiência Matemática. Lisboa: Gradiva, 2013.

FIORENTINI, D. Alguns Modos e ver e conceber o ensino da matemática no Brasil. Zetetiké, v. 3, n. 4, p.1-37, 1995.

FIORENTINI, D.; OLIVEIRA, A. O Lugar das Matemáticas na Licenciatura em Matemática: que matemáticas e que práticas formativas? Bolema, v. 27, n. 47, p. 917-938, 2013.

KILPATRICK, J. A, Higher Standpoint. Proceedings of the $11^{\text {th }}$ International Conference of Mathematical Education. México, 2008.

KLEIN, F. Elementary Mathematics from an Advanced Standpoint: Arithmetic, Algebra, Analysis. USA: Dover, 2004.

MIGUEL, A.; VILELA, D. Práticas escolares de mobilização de cultura matemática. Cadernos CEDES, v. 28, n. 74, p. 97-120, 2008.

MOREIRA, P.; FERREIRA, A. O Lugar da Matemática na Licenciatura em Matemática. Bolema, v. 27, n. 47, p. 981-1005, 2013.

RANGEL, L.; GIRALDO, V.; MACULAN, N. Conhecimento de matemática para o ensino: um estudo colaborativo sobre números racionais. Jornal Internacional de Estudos em Educação Matemática, v. 8, p. 42-70, 2015.

SHULMAN, L. Those who understand: Knowledge growth in teaching. Educational Researcher, v.15, p. 4-14, 1986. 


\section{Anexo: Enunciados das Tarefas}

Figura 3: Tarefa 1.

$\underline{\text { Tarefa } 1}$ - Em uma avaliação aplicada no Ensino Médio, o aluno deveria resolver a seguinte questão:

Dada a função $f: \mathbb{R} \rightarrow \mathbb{R}$ tal que $f(x)=\left\{\begin{array}{l}2 x^{4}+3 x^{3}-5 x^{2}-9 x-3 ; x<1 \\ x^{6}+x^{4}+1 ; \quad x \geq 1\end{array}\right.$, é correto afirmar que:

a) $f$ possui pelo menos um zero no intervalo $] 0,2[$ ?

b) $f$ possui exatamente um zero no intervalo $]-2,0[$ ?

Dois alunos responderam da seguinte maneira:

\section{Solução de Ana}

\section{a) $f(0)=-3$}

$f(2)=2^{6}+2^{4}+1=81$

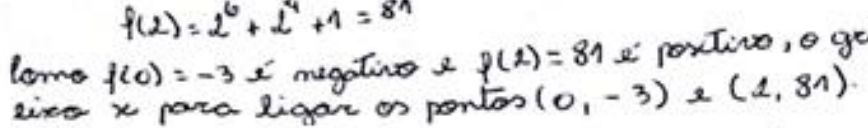

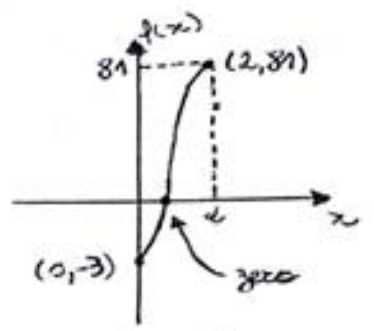

Assion, if turá pelo menas um zeco em $] O, 2[$

b) Xssim, como foi feito no item anterior, temos

$f(0)=-3$

$f(-2)=2 \cdot(-2)^{4}+3 \cdot(-2)^{3}-5 \cdot(-2)^{2}-9 \cdot(-2)-3=3$

lome $f(0)=-3$ i megativo e $f(-2)=3$ i pontivo, a gráfico de $f$ iré coetar a uxo $x$ pora ligar as portes $(0,-3)=(-2,3)$.

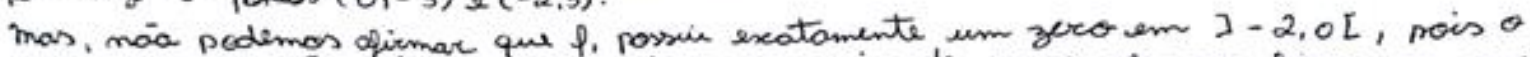
grafica da função pode cortar o lixo $x$ mais de uma vey para ligar as pon $\tan (0,-3)$ i $(-2,3)$

ló podimas afirmar, com extena, que o gráfico cota o eixo $x$ em ume quantidade impar de vezes para ligar estes rontas.
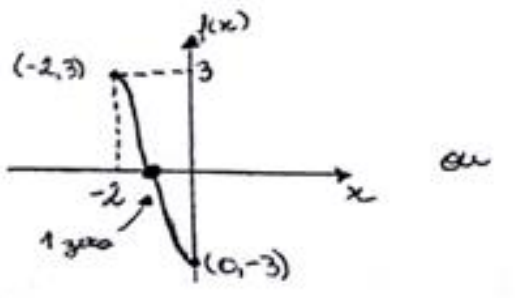

$1,3,5, \ldots$ (nimero impor de zeros)

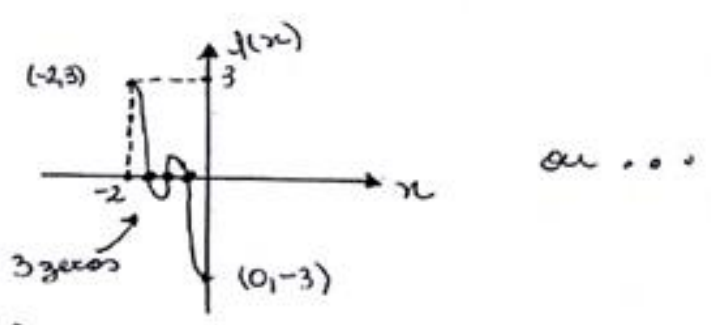

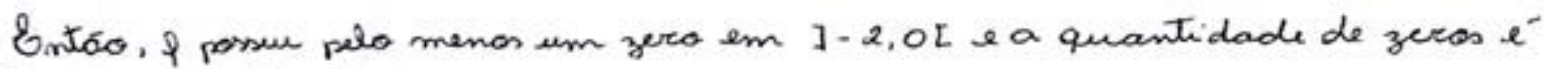
impar messe intervalo 


\section{Solução de Carlos}

a) Casa $x \geqslant 1$, temos que $x^{6}, x^{4} \geqslant 1 . \operatorname{tog} \theta, f(x) \geqslant 3 a$, portantio, f noio possui zero em $[1,2 L$.

- Caso $0<x<1$, temos que encontrar as ravizes do. polinámia $p(x)=2 x^{4}+3 x^{3}-5 x^{2}-9 x-3$.

$a= \pm 1 ; \pm 3$ sáo es divisores de $a_{0}=-3$

$b= \pm 1 ; \pm 2$ sāo os divisares de $a_{4}=2$

As passíveis raizes reais do palinámio sāo da forma $\frac{a}{b}$.

Candidatos a rózes: $\pm 1 ; \pm 3 ; \pm \frac{1}{2} ; \pm \frac{3}{2}$ De todos os candidatos, apenas -1 e $-\frac{1}{2}$ sä́ racizes do polinómia, pais $p(-1)=p\left(-\frac{1}{2}\right)=0.2 \operatorname{tog} \theta, *$ Tambein nāo possui zera em $] 0,1[$. Assim, f naio passui zero em $70,2[$.

\section{zeros da punção para $x<1$. Assim, f possui exatamente dois zeros em}

\section{Questões}

I) Resolva o exercício que foi proposto.

II) Em sua opinião, qual o objetivo do exercício acima?

III) Analise as soluções dos alunos, fazendo comentários sobre os argumentos apresentados por eles em suas respostas.

IV) Qual o retomo que você daria aos alunos de acordo com as soluções apresentadas para o exercício?

V) Você identifica alguma relação entre esta tarefa (incluindo as soluções apresentadas) e o conteúdo de Análise? Em caso afirmativo, cite exemplos de associações que podem ser feitas.

Fonte: Elaborada pelos autores. 


\section{Figura 4: Tarefa 2.}

\section{$\underline{\text { Tarefa } 2}$}

Classifique em Verdadeiro (V) ou Falso (F) as afirmações abaixo, justificando suas respostas.

a) Seja $f:[-1,1] \rightarrow \mathbb{R}$ uma função descontínua em algum $x_{0} \in[-1,1]$. Se $f(-1)<0<f(1)$, então existe $c \in]-1,1[$ tal que $f(c)=0$.

b) Seja $f:[-1,1] \rightarrow \mathbb{R}$ uma função descontínua em algum $x_{0} \in[-1,1]$. Se $f(-1)<0<f(1)$, então não existe $c \in]-1,1[$ tal que $f(c)=0$.

c) Seja $f:[-1,1] \rightarrow \mathbb{R}$ contínua. Se $f(-1)<0<f(1)$, então existe $c \in]-1,1[$ tal que $f(c)=0$.

d) Seja $f:[-1,1] \rightarrow \mathbb{R}$ uma função contínua. Se $f(-1)<0<f(1)$, então existe um único $c \in]-1,1[$ tal que $f(c)=0$.

e) Seja $f:\left[-1, \frac{1}{3}\right] \cup\left[\frac{2}{3}, 1\right] \rightarrow \mathbb{R}$ uma função contínua. Se $f(-1)<0<f(1)$, então existe $\left.c \in]-1, \frac{1}{3}\right] \cup\left[\frac{2}{3}, 1[\right.$ tal que $f(c)=0$.

Fonte: Elaborada pelos autores.

Figura 5: Tarefa 3.

$\underline{\text { Tarefa } 3}$ - (Teorema do Valor Intermediário) Seja $f:[a, b] \rightarrow \mathbb{R}$ contínua. Se $f(a)<k<f(b)$, então existe $c \in] a, b[$ tal que $f(c)=k$.

Dois alunos de um curso de Análise demonstraram o teorema da seguinte maneira:

Demonstração de Pedro: Seja $A=\{x \in[a, b] ; f(x) \leq k\}$. O conjunto $A$ é não vazio (pois $a \in A$ ) e limitado superiormente ( $b$ é cota superior de $A$, uma vez que $b \notin A$ ). Logo, existe $c=\sup A$.

Assim, existe uma sequencia $\left(x_{n}\right) \subset A$ tal que $\lim x_{n}=c$. Como $f$ é contínua, temos que $\lim f\left(x_{n}\right)=f(c)$. Segue, também, que $f\left(x_{n}\right) \leq k$ para todo $n \in \mathbb{N}$, uma vez que $\left(x_{n}\right) \subset A$. Portanto, $f(c) \leq k$ e, consequentemente, $c<b$.

Provemos, agora, que $f(c)=k$. Suponha, por absurdo, que $f(c)<k$. Como $f$ é contínua, existe $\delta>0$ tal que para todo $x \in[a, b]$ e $|x-c|<\delta$ temos $f(x)<k$. Logo, existe $x \in[a, b] \operatorname{com} c<x<c+\delta$ tal que $f(x)<k$. Portanto, $x \in A$ e $x>c=\sup A$. Porém, isso representaria uma contradição, pois existiria um elemento do conjunto $A$ maior que o supremo. Então, $f(c)=k$. 
Demonstração de Júlia: Dividimos o intervalo $[a, b]$ ao meio, obtendo dois novos intervalos da seguinte maneira: caso $f\left(\frac{a+b}{2}\right)=k$, o teorema está provado; caso $f\left(\frac{a+b}{2}\right)<k$, então tome $a_{1}=\frac{a+b}{2}$ e $b_{1}=b$; caso $f\left(\frac{a+b}{2}\right)>k$, considere $a_{1}=a$ e $b_{1}=\frac{a+b}{2}$.

$$
\begin{aligned}
& \text { - Caso } f\left(\frac{a+b}{2}\right)<k \\
& \alpha_{1}=\frac{a+b}{2} \\
& b_{1}=b \\
& I_{1}=\left[a_{1}, b_{1}\right]
\end{aligned}
$$

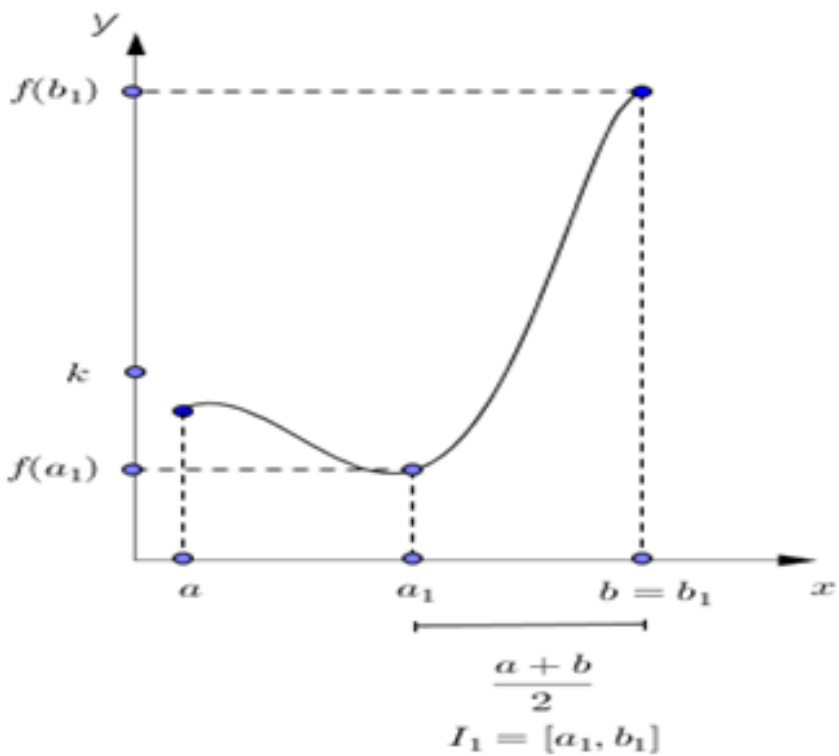

Logo, obtemos um novo intervalo $I_{1}=\left[a_{1}, b_{1}\right] \subset[a, b]$ de comprimento $\frac{a+b}{2}$ tal que $f\left(a_{1}\right) \leq k \leq f\left(b_{1}\right)$.

Novamente, dividimos $I_{1}$ ao meio e repetimos o processo analisando $f\left(\frac{a_{1}+b_{1}}{2}\right)$. Desta forma, determinamos um intervalo $I_{2}=\left[a_{2}, b_{2}\right] \subset I_{1} \subset[a, b]$ com comprimento $\frac{a+b}{4}$.

$$
\begin{aligned}
& \text { - Caso } f\left(\frac{a_{1}+b_{1}}{2}\right)>k \\
& \alpha_{2}=\alpha_{1} \\
& b_{2}=\frac{a_{1}+b_{1}}{2} \\
& I_{2}=\left[a_{2}, b_{2}\right]
\end{aligned}
$$

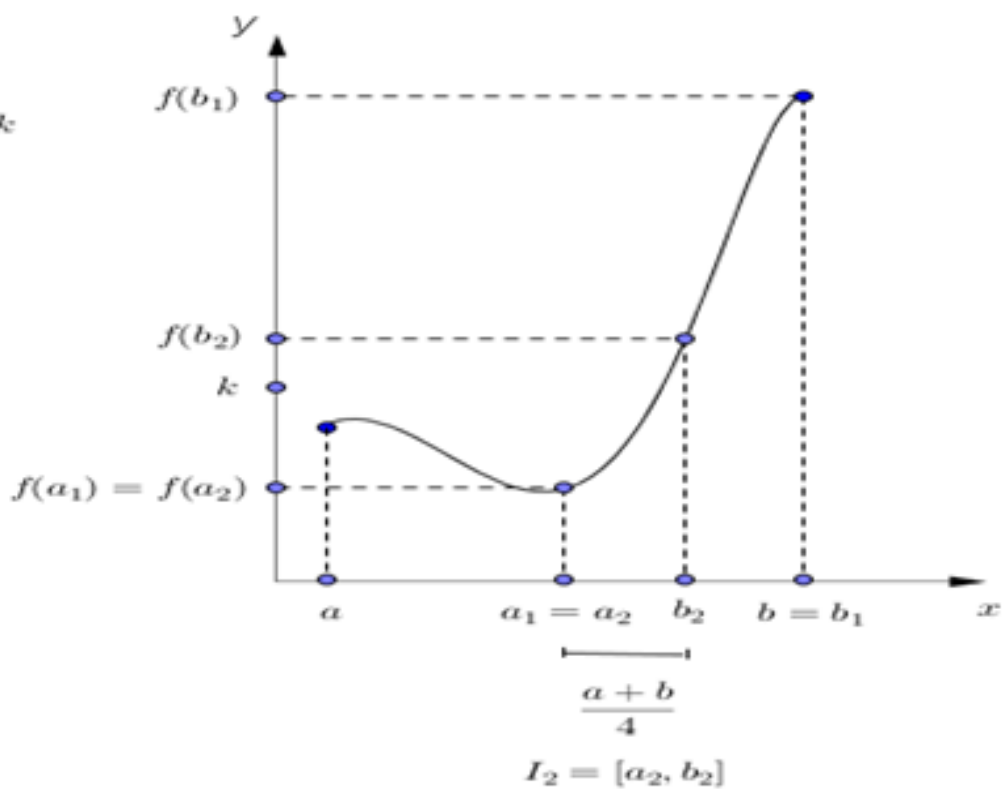


Prosseguindo desta maneira sucessivamente, obtemos uma sequência de intervalos encaixados $[a, b]=I_{0} \supset I_{1} \supset I_{2} \supset \cdots$, onde $I_{n}=\left[a_{n}, b_{n}\right]$ tem comprimento $\frac{a+b}{2^{n}}$.

Portanto, pelo teorema dos intervalos encaixados, existe $c \in \cap I_{n}$. Como o comprimento dos intervalos tende à zero, esta intersecção se reduz exclusivamente ao valor $c$. Temos ainda que $c=\lim a_{n}=\lim b_{n}$

Assim, como $f$ é contínua em $c$ e $f\left(a_{n}\right) \leq k \leq f\left(b_{n}\right)$, temos que $f(c)=\lim f\left(a_{n}\right) \leq k \leq \lim f\left(b_{n}\right)=f(c)$. Logo, $f(c)=k$.

\section{Questões}

I) Você considera corretas as duas demonstrações apresentadas? Discuta a validade das demonstrações analisando os argumentos apresentados.

II) Qual o retomo que você daria aos alunos de acordo com as demonstrações apresentadas para o Teorema do Valor Intermediário?

III) Em sua opinião, qual das duas demonstrações permite compreender melhor os argumentos utilizados? Justifique sua resposta.

IV) Em sua opinião, qual das duas demonstrações permite compreender melhor o significado do enunciado do teorema? Justifique sua resposta.

Fonte: Elaborada pelos autores.

Submetido em janeiro de 2017 Aprovado em março de 2017 